$=-R T 2,3026 \log K$ wurden die G-Werte ermittelt, die in Tab. 3 mitaufgeführt sind. Nach $\Delta G-\Delta H=$ $-T \Delta S$ ergibt sich die Reaktionsentropie $\Delta \mathrm{S}=21,20 \mathrm{Cl}$, während nach

$$
\left(\frac{\partial G}{\partial T}\right)_{p}=-\Delta S
$$

derselbe Wert 21,20 erhalten wurde. Mit diesen Werten läßt sich die Beziehung

$$
\log K=-1082 / T+4,634
$$

zwischen $K$ und $1 / T$ aufstellen.

\title{
Die Wärmetönung der Bildung von Natriumoxalat aus Natriumformiat
}

\author{
Von G.-M. Schwab*, S. Paparos und I. Tsipouris \\ Aus dem Physikal.-Chem. Laboratorium der Technischen Hochschule Athen \\ und dem Physikal.-Chem. Institut der Universität München \\ (Z. Naturforschg. 6 a, 387-390 [1951]; eingegangen am 23. April 1951)
}

\begin{abstract}
Herrn Prof. John Eggert, dem bewährten Lehrer ganzer Generationen von Physikochemikern und dem hochverdienten Forscher auf dem Gebiete der reinen und angewandten Photochemie, sei dieser Beitrag mit den herzlichsten Wünschen für sein weiteres Wirken zum 60. Geburtstag in Freundschaft gewidmet.
\end{abstract}

Es wird die Wärmetönung der Zersetzung von Natriumformiat in Natriumoxalat und Wasserstoff auf indirektem Wege bestimmt, indem kalorimetrisch die Umsetzungen von Natriumformiat einerseits und Natriumoxalat andererseits mit konz. Schwefelsäure verfolgt werden. Die Kombination dieser beiden Wärmetönungen mit derjenigen der Wassergasreaktion ergibt, daß die Umsetzung von festem Formiat zu Oxalat mit 4 kcal je Mol Oxalat endotherm ist, daß aber die technische Zersetzung von geschmolzenem Formiat um etwa ebensoviel exotherm ist. Das Ergebnis wird diskutiert.

$\mathrm{B}$ ei der technischen Herstellung von Natriumoxalat aus Natriumformiat nach

$$
2 \mathrm{NaOOCH} \longrightarrow \mathrm{Na}_{2} \mathrm{C}_{2} \mathrm{O}_{4}+\mathrm{H}_{2}+Q
$$

wird die Wärme $Q$ frei, die schon im Laboratorium und noch mehr beim Arbeiten im technischen Maßstab eine starke Selbsterhitzung der geschmolzenen Reaktionsmasse bei fast explosionsartigem Reaktionsablauf mit sich bringt. Für das Studium dieser merkwürdigen Reaktion und der sie begleitenden Nebenreaktion

$$
2 \mathrm{NaOOCH} \longrightarrow \mathrm{Na}_{2} \mathrm{CO}_{3}+\mathrm{CO}+\mathrm{H}_{2}
$$

sowie der noch merkwürdigeren katalytischen Einflüsse auf beide war die Kenntnis der Wärmetönung $Q$ wünschenswert. Für die Bildungswärmen des Natriumformiats und -oxalats aus den Elementen im Normalzustand finden sich folgende Literaturangaben:

\begin{tabular}{lcr} 
& Berthollet $^{1}$ & Lange $^{2}$ \\
$2 \mathrm{NaOOCH}$ & 318 & 314,02 \\
$\mathrm{Na}_{2} \mathrm{C}_{2} \mathrm{O}_{4}$ & 315 & 316,01 \\
\hline$Q$ & -3 & $+1,99$
\end{tabular}

* München 2, Sophienstr. 11.

1 M. B e r t h o l le t, Thermochimie, Paris 1897, II, 215 (Aus Verbrennungs- und Neutralisationswärmen; M. B e r th o lle t, Ann. Chim. physique (5) 4, 108 [1875]; 6, 326 [1875]).
Demnach ist nicht einmal sicher, ob die Reaktion von festem Natriumformiat zu festem Oxalat endotherm oder exotherm ist. Es erschien daher zweckmäßig, die Wärmetönung erneut zu bestimmen. Eine unmittelbare kalorimetrische Messung kommt nicht in Frage, weil die Reaktion (1) in wechselndem Umfang von (2) begleitet ist und überdies nicht immer quantitativ vollständig verläuft, abgesehen von den hohen erforderlichen Temperaturen. Es wurde daher vorgezogen, die Differenz der Umsetzungwärmen der beiden Salze mit konz. Schwefelsäure zu bestimmen nach dem Schema:

$$
2 \mathrm{NaOOCH}+\mathrm{H}_{2} \mathrm{SO}_{4} \rightarrow \mathrm{Na}_{2} \mathrm{SO}_{4}+2 \mathrm{CO}+2 \mathrm{H}_{2} \mathrm{O}(\mathrm{aq})+Q_{1}
$$

$\mathrm{Na}_{2} \mathrm{C}_{2} \mathrm{O}_{4}+\mathrm{H}_{2} \mathrm{SO}_{4} \rightarrow \mathrm{Na}_{2} \mathrm{SO}_{4}+\mathrm{CO}+\mathrm{CO}_{2}+\mathrm{H}_{2} \mathrm{O}(\mathrm{aq})+Q_{2}$

und das Ergebnis mit der bekannten Wassergasreaktion zu kombinieren:

$$
\mathrm{CO}_{2}+\mathrm{H}_{2} \rightarrow \mathrm{H}_{2} \mathrm{O}(\mathrm{aq})+\mathrm{CO}+Q_{3},
$$

2 N. A. L a n g e, Handbook of Chemistry, Cleveland 1932. 
was zu dem gewünschten Ergebnis führt:

$$
2 \mathrm{NaOOCH} \longrightarrow \mathrm{Na}_{2} \mathrm{C}_{2} \mathrm{O}_{4}+\mathrm{H}_{2}+\left(Q_{1}-Q_{2}-Q_{3}\right) \text {. }
$$

Die Reaktionen (3) und (4) gehen bei Temperaturen von $120^{\circ}-150^{\circ} \mathrm{C}$ in konz. Schwefelsäure mit solcher Geschwindigkeit vor sich, daß sie bequem kalorimetrisch meßbar sind.

Zwei Korrekturen sind dabei zu beachten:

a) Es entstehen Natriumsulfat und Wasser nicht in freier Form, sondern gelöst in konz. Schwefelsäure. Was das Natriumsulfat betrifft, so wurde einfach so verfahren, daß immer äquivalente Mengen von Formiat bzw. Oxalat verwandt wurden, so daß die entstandene Sulfatlösung stets etwa dieselbe Konzentration hatte. Hierdurch hebt sich in $Q_{1}-Q_{2}$ die Lösungswärme des Natriumsulfats heraus. Das gleiche ist nicht gleichzeitig für das Wasser möglich, weil in (3) doppelt soviel Wasser entsteht wie in (4). Die Verdünnungswärme eines Mols Schwefelsäure durch $x$ Mole Wasser beträgt nach $\mathrm{T}$ h o m p s o $\mathrm{n}^{3}$ :

$$
W=\frac{17860 x}{x+1,798} \mathrm{cal},
$$

und daher ist die Lösungswärme eines Mols Wasser in einer anfänglichen azeotropen Schwefelsäure von $x_{0}=0,09415 \mathrm{Mol} / \mathrm{Mol}$ (gleich $98,3 \%{ }^{4}$ ) bis zum Molenbruch $x_{0}+x$ :

$$
W=\frac{17860\left(x_{0}+x\right)}{\left(x_{0}+x+1,798\right) x}-\frac{17860 x_{0}}{\left(x_{0}+1,798\right) x},
$$

wobei sich $x$ aus der Menge vorgelegter Schwefelsäure (etwa $130 \mathrm{~cm}^{3}$ ) und des eingewogenen Salzes (etwa 1,3 g) ergibt. Es wird dabei mangels eingehenderer Daten vorausgesetzt, daß diese Verdünnungswärme sich nicht wesentlich mit der Temperatur verändert.

b) Es erwies sich aus praktischen Gründen wegen der Heftigkeit der Reaktionen nicht als durchführbar, die Salze schon während der Vorperiode im Kalorimeter zu temperieren und dann zur Reaktion zu bringen, vielmehr mußten sie im kalten Zustand als $\mathrm{Pa}-$ stillen in die heiße Schwefelsäure eingeworfen werden. Hierbei nehmen sie Wärme

$$
H=n \int_{T_{1}}^{T} C_{p} \mathrm{~d} T
$$

2 Landolt-Börnstein, Physik.-chem. Tab., 2. Erg.-Bd., 1548.

${ }^{4}$ Landolt-Börnstein, Physik.-chem. Tab., Bd. 2, 1833; 1. Erg.-Bd., 792. auf. $\left(T_{1}=\right.$ Zimmertemperatur, $T=$ Versuchstemperatur, $n=$ Molzahl des Salzes, $C_{\mathrm{p}}=$ Molwärme des Salzes unter konstantem Druck.) In der Literatur ${ }^{5}$ sind die Angaben über die hier interessierenden spezif. Wärmen höchst lückenhaft und müssen durch Abschätzungen ergänzt werden. Für Natriumformiat werden zwei um $6 \%$ differierende Werte und kein Temperaturkoeffizient angegeben. Wir helfen uns durch Einführung eines Mittelwertes und die Annahme, daß der relative Temperaturkoeffizient $\frac{1}{C} \frac{\mathrm{d} C}{\mathrm{~d} T}$ derselbe ist wie der angebbare des Natriumchlorats sowie daß $\begin{aligned} & \mathrm{d} C \\ & \mathrm{~d} T\end{aligned}$ für alle Salze temperaturunabhängig ist. Diese Annahmen führen auf:

$$
C_{\mathrm{NaOOCH}}=19,54+0,0217 t
$$

\section{( $t=$ Celsius-Temperatur).}

Die ebenfalls unbekannte spezif. Wärme des Natriumoxalats berechnen wir nach der Neumann-Koppschen Regel durch Abzug des Wertes zweier Wasserstoffatome von dem doppelten des obigen Wertes. Wiederum mit der Annahme eines gemeinsamen relativen Temperaturkoeffizienten erhalten wir:

$$
C_{\mathrm{Na}_{2} \mathrm{C}_{2} \mathrm{O}_{4}}=34,48+0,0386 t .
$$

Bezeichnen wir nun mit $Q_{1}^{\prime}$ die bei der Reaktion (1) experimentell beobachtete Wärme und mit $Q_{1}{ }^{\prime \prime}$ die Verdünnungswärme der Schwefelsäure, beide je $2 \mathrm{Mol}$ Salz, so wird

$$
Q_{1}=Q_{1}{ }^{\prime}+H_{1}-Q_{1}^{\prime \prime}
$$

und entsprechend $Q_{2}=Q_{2}{ }^{\prime}+H_{2}-Q_{2}{ }^{\prime \prime}$, wobei $Q_{2}{ }^{\prime \prime}$ sich auf 1 Mol Wasser bezieht.

$$
\text { Versuchsanordnung }
$$

Die Aufgabe, kalorimetrische Messungen in konz. Schwefelsäure der Temperatur $130-150^{\circ} \mathrm{C}$ auszuführen, läßt sich unter Verwendung eines Dewar-Bechers als Kalorimetergefäß lösen. Es wurde ein versilberter Becher von $6 \mathrm{~cm}$ lichtem Durchmesser und $15 \mathrm{~cm}$ lichter Höhe gewählt, der mit einem dicken Kautschukstopfen verschlossen war. Der Stopfen trug Bohrungen für einen elektrisch betätigten Glasrührer, ein Beckmann-Thermometer mit $1 /{ }_{100}{ }^{\circ}$ Teilung, ein gewöhnliches Thermometer, einen in Glas montierten elektrischen Heizer von etwa $3 \mathrm{Ohm}$ Widerstand sowie eine verschließbare Bohrung für den Einwurf der Substanz. Die elektrische Heizung erfolgte zwecks Erreichung der Versuchstemperatur vor dem Versuch mit Wechselstrom, bei der Eichung des Wasserwertes des Kalorimeters mit Gleichstrom gemessener Spannung und Stromstärke. Dieser wurde aus einer Akkumulatoren-

5 Gmelin, Handbuch der anorg. Chemie, 8. Aufl., Bd. Natrium. 


\begin{tabular}{|c|c|c|c|c|c|c|c|c|c|c|c|c|c|}
\hline $\begin{array}{l}\text { Vers.- } \\
\text { Nr. }\end{array}$ & $\begin{array}{l}\text { Tempe- } \\
\text { ratur }\end{array}$ & $\begin{array}{l}\text { Sub- } \\
\text { stanz }\end{array}$ & $\underset{\mathrm{g}}{\text { Menge }}$ & $\begin{array}{l}\text { Wärme- } \\
\text { kapa- } \\
\text { zität }\end{array}$ & \lrcorner $T$ & $\begin{array}{c}Q_{1}^{\prime} \\
\mathrm{kcal} / \mathrm{Mol} \\
\mathrm{Na}_{2}\end{array}$ & $\begin{array}{c}H_{1} \\
\mathrm{kcal} / \mathrm{Mol} \\
\mathrm{Na}_{2} \mathrm{SO}_{4}\end{array}$ & $Q_{1}{ }^{\prime \prime}$ & $Q_{1}$ & $\begin{array}{c}Q_{1} \\
\text { Mittel- } \\
\text { wert }\end{array}$ & $\begin{array}{c}\text { Gesamt- } \\
\text { mittel }{ }^{*} \\
\left(140^{\circ}\right)\end{array}$ & $\begin{array}{c}Q_{3} \\
\left(140^{\circ}\right)\end{array}$ & $Q$ \\
\hline 1 & 150 & $\mathrm{NaO}_{2} \mathrm{CH}$ & 1,4079 & 139,15 & 2,60 & 34,95 & 5,56 & 17,86 & 22,65 & & \multirow{7}{*}{21,5} & \multirow{7}{*}{$-0,6$} & \\
\hline 2 & 150 & $\mathrm{NaO}_{2} \mathrm{CH}$ & 1,5106 & 140,27 & 2,81 & 35,49 & 5,56 & 17,75 & 23,30 & $22,6 \pm 1$ & & & \\
\hline 3 & 150 & $\mathrm{NaO}_{2} \mathrm{CH}$ & 1,2857 & 137,36 & 2,40 & 34,87 & 5,56 & 17,87 & 22,57 & & & & $-26,5$ \\
\hline 4 & 130 & $\mathrm{NaO}_{2} \mathrm{CH}$ & 1,2101 & 125,76 & 2,40 & 33,92 & 4,38 & 17,87 & 20,43 & \multirow{3}{*}{$20,4 \pm 1$} & & & $\begin{array}{l}+\quad 0,0 \\
\end{array}$ \\
\hline 5 & 130 & $\mathrm{NaO}_{2} \mathrm{CH}$ & 1,2761 & 123,81 & 2,57 & 33,91 & 4,40 & 17,86 & 20,44 & & & & $-\begin{array}{c}4,4 \\
\mathrm{kcal}\end{array}$ \\
\hline 6 & 130 & $\mathrm{NaO}_{2} \mathrm{CH}$ & 1,2387 & 120,59 & 2,556 & 33,84 & 4,38 & 17,87 & 20,35 & & & & $\begin{array}{r}\text { kcal } \\
\pm \quad 2\end{array}$ \\
\hline & & & & & & $Q_{2}^{\prime}$ & $H_{2}$ & $Q_{2}{ }^{\prime \prime}$ & $Q_{2}$ & $\underset{\text { Mittel }}{Q_{2}}$ & & & \\
\hline 7 & 130 & $\mathrm{Na}_{2} \mathrm{C}_{2} \mathrm{O}_{4} \mid$ & 1,4084 & 128,4 & 2,73 & 33,35 & 3,90 & 10,2 & 27,05 & & \multirow{5}{*}{26,5} & & \\
\hline 8 & 130 & $\mathrm{Na}_{2} \mathrm{C}_{2} \mathrm{O}_{4}$ & 1,2310 & 112,9 & 2,26 & 32,09 & 3,90 & 10,2 & 25,79 & $26,7 \pm 1$ & & & \\
\hline 9 & 130 & $\mathrm{Na}_{2} \mathrm{C}_{2} \mathrm{O}_{4}$ & 1,2838 & 131,15 & 2,44 & 33,40 & 3,90 & 10,2 & 27,10 & & & & \\
\hline 10 & 150 & $\mathrm{Na}_{2} \mathrm{C}_{2} \mathrm{O}_{4}$ & 1,3026 & 131,03 & 2,38 & 32,08 & 4,73 & 10,2 & 26,61 & \multirow{2}{*}{$26,3 \pm 1$} & & & \\
\hline 11 & 150 & $\mathrm{Na}_{2} \mathrm{C}_{2} \mathrm{O}_{4}$ & 1,3266 & 128,44 & 2,43 & 31,53 & 4,73 & 10,2 & 26,06 & & & & \\
\hline
\end{tabular}

* Die Differenz der für $150^{\circ}$ und $130^{\circ}$ gefundenen Werte für Formiat ist nicht durch irgendwelche wahrscheinlichen Werte der spezif.Wärmen der Teilnehmer erklärbar, und da diese bei Natriumoxalat praktisch nicht auftritt, so ist sie eher auf Versuchsfehler zurückzuführen als etwa auf eine Temperaturabhängigkeit der Verdünnungswärme der Schwefelsäure. Es scheint daher gerechtfertigt, ein "Gesamtmittel“" für die mittlere Temperatur von $140^{\circ} \mathrm{C}$ aufzustellen.

Tab. 1.

batterie von 6 Volt entnommen, die während des Nichtgebrauchs über einen dem Heizer gleichen Widerstand geschlossen blieb.

\section{Arbeitsweis e}

Das Kalorimeter wird mit etwa $130 \mathrm{~cm}^{3}$ azeotroper Schwefelsäure (s. oben) beschickt und verschlossen. Es erwies sich als notwendig, für jeden Versuch frische Säure zu verwenden, weil sonst wegen der veränderten Massen und Lösungswärmen die Reaktionswärmen bei mehrmaliger Verwendung derselben Säure einen fallenden und die Wärmekapazitäten einen steigenden Gang aufwiesen. Die Flüssigkeit wird sodann mit Wechselstrom auf die beabsichtigte Versuchstemperatur aufgeheizt, das BeckmannThermometer eingestellt und an diesem die Abkühlungskurve beobachtet. Diese ist wegen der Einfachheit unserer Anordnung relativ steil (etwa $0,7^{\circ}$ pro Min. bei $150^{\circ}$ und etwa $0,55^{\circ}$ pro Min. bei $130^{\circ}$ ), jedoch wegen des im Vergleich zu dem ganzen Temperaturunterschied sehr geringen Beobachtungsintervalles vollkommen geradlinig und auch für die Vor- und Nachperiode von Eichung und Messung vollkommen parallel. Sodann wird mit gemessenem Gleichstrom von etwa 1,1 Amp. 2-3 Min. lang wieder um einige Grade aufgeheizt, erneut die Abkühlungsgerade beobachtet, sodann die Substanz eingeworfen und nach Beendigung der Erwärmung wiederum die Abkühlungsgerade gemessen. Schließlich wird nochmals elektrisch geheizt und eine letzte Abkühlungsgerade aufgenommen. Wegen der Parallelität der Abkühlungsgeraden konnte die der Heizenergie bzw. der Reaktionswärme entsprechende Temperaturerhöhung einfach direkt aus dem Temperaturabstand der betreffenden beiden Geraden entnommen werden. Zur Berechnung der Reaktionswärme wurde der Mittelwert der vor und nach der Reaktion bestimmten Wärmekapazitäten eingesetzt.

\section{Meßergebnis se}

Die Ergebnisse der Messungen sind in der Tab. 1 zusammengestellt. Es ergibt sich, daß die Bildung.von gasförmigem Kohlenoxyd, gelöstem Natriumsulfat und freiem flüssigem Wasser aus $2 \mathrm{Mol}$ festem Natriumformiat $21,5 \mathrm{kcal}$ entwickeln würde, während die Bildung von gasförmigem Kohlenmonoxyd und Kohlendioxyd, gelöstem Natriumsulfat und freiem flüssigem Wasser aus einem Mol Natriumoxalat 26,5 kcal entwickeln würde. Diese Zahlen beziehen sich auf die mittlere Temperatur sämtlicher Versuche, also auf $140^{\circ} \mathrm{C}$.

\section{Die Wärmetönung der Wassergas - reaktion}

Es bleibt uns noch, die Wärmetönung der Wassergasreaktion unter Bildung von flüssigem Wasser bei $140^{\circ} \mathrm{zu}$ berechnen. Wir gehen dazu aus von der für $25^{\circ}$ und gasförmiges Wasser geltenden thermochemischen Gleichung ${ }^{6}$

$$
\mathrm{CO}+\mathrm{H}_{2} \mathrm{O}_{\mathrm{g}} \rightarrow \mathrm{CO}_{2}+\mathrm{H}_{2}+9,81 \mathrm{kcal}
$$

und benutzen folgende Ausdrücke für die spezif. Wärmen $C_{\mathrm{p}}$ der Teilnehmer ${ }^{7}$ :

3 A. R. Gordo n, J. chem. Physics 1, 308 [1933]; Landolt-Börnstein, Physik.-chem. Tab., 3. Erg.-Bd., 2749.

$7 \mathrm{Ke} l 1 \mathrm{y}$, in: Landolt-Börnstein, Physik.-chem. Tab., 3. Erg.-Bd., 2326. 


$$
\begin{array}{rr}
\mathrm{CO}: & 6,60+1,20 \cdot 10^{-3} T \\
\mathrm{CO}_{2}: & 10,34+2,74 \cdot 10^{-3} T-1,955 \cdot 10^{-5} T^{2} \\
\mathrm{H}_{2}: & 6,62+0,81 \cdot 10^{-3} T \\
\mathrm{H}_{2} \mathrm{O}_{\mathrm{g}}: & 8,22+0,15 \cdot 10^{-3} T+1,34 \cdot 10^{-6} T^{2}
\end{array}
$$

Wir erhalten so in bekannter Weise für die Temperatur von $140^{\circ} \mathrm{C}$ :

$$
\mathrm{CO}+\mathrm{H}_{2} \mathrm{Og} \longrightarrow \mathrm{CO}_{2}+\mathrm{H}_{2}+9,78 \mathrm{kcal}
$$

und nach Abzug der Verdampfungswärme des Wassers bei $140^{\circ} 8$ von $9,206 \mathrm{kcal} / \mathrm{Mol}$ :

oder

$$
\mathrm{CO}+\mathrm{H}_{2} \mathrm{Ofl} \longrightarrow \mathrm{CO}_{2}+\mathrm{H}_{2}+0,6 \mathrm{kcal}
$$

$$
Q_{3}=-0,6 \mathrm{kcal} / \mathrm{Mol} .
$$

Gesamtergebnis und Besprechung

Wir erhalten so für die bei der Umsetzung von 2 Mol Natriumformiat zu einem Mol Natriumoxalat freiwerdende Wärme $Q$ :

$Q=Q_{1}-Q_{2}-Q_{3}=21,5-26,5+0,6=-4,4 \mathrm{kcal} / \mathrm{Mol}$.

Das bedeutet, daß die genannte Umsetzung bei $140^{\circ}$ und ausgehend von festem Formiat endotherm ist, in qualitativer Übereinstimmung mit den älteren thermochemischen Daten ${ }^{1}$ und im Gegensatz zu den neue- . ren ${ }^{2}$. Die Genauigkeit unseres Ergebnisses ist nach Prüfung aller Fehlergrenzen auf \pm 2 kcal zu veranschlagen. Dieses für die Einfachheit der Methoden relativ günstige Ergebnis kommt daher, daß das Endresultat die Differenz zweier im Vergleich zu den Bildungswärmen recht kleiner Wärmetönungen ist. Im

8 W.Schüle, Z. Ver. dtsch. Ing. 55/II, 1506 [1911]; Landolt-Börnstein, Physik.-chem. Tab., 1. Erg.-Bd., 863.
Rahmen dieser Fehlergrenze erscheint der endotherme Charakter der Reaktion eben noch sichergestellt.

Unser Ergebnis steht selbstverständlich nicht im Gegensatz zu der allgemeinen Betriebserfahrung, daß die fragliche Umsetzung unter heftiger Wärmeentwicklung verläuft. In der Praxis wird nämlich geschmolzenes Natriumformiat zu festem Oxalat zersetzt, wobei zusätzlich die Schmelzwärme von $2 \mathrm{Mol}$ Natriumformiat frei wird. Nun ist leider die Schmelzwärme des Natriumformiats nicht bekannt. Wir berechnen jedoch aus den Schmelzwärmen und Schmelzpunkten von 8 anderen Natriumsalzen, die in Gmelins Handbuch aufgeführt sind, einen Mittelwert der Schmelzentropie von $7,4 \pm 1,7$ Clausius und daraus mit dem Schmelzpunkt des Natriumformiats von $255^{\circ} \mathrm{C}$ eine Schmelzwärme von $4 \pm 1 \mathrm{kcal} / \mathrm{Mol}$. Die technische Reaktion wird dann:

$$
2 \mathrm{NaOOCH}_{\mathrm{fl}} \longrightarrow \mathrm{Na}_{2} \mathrm{C}_{2} \mathrm{O}_{4} \text { fest }+\mathrm{H}_{2}+(4 \pm 3) \mathrm{kcal} .
$$

Sie ist also gerade noch mit Sicherheit exotherm. Es ist bemerkenswert, daß die starke Selbsterhitzung des Reaktionsgutes, die in allen Versuchsanordnungen beobachtet wird, hiernach nicht etwa auf einer besonders hohen Wärmeentwicklung beruht, sondern vielmehr auf dem kurzen Zeitintervall der Reaktion, die auch bei großen Ansätzen in wenigen Minuten beendet ist. Man wird daraus schließen dürfen, daß es sich nicht um eine einfache „Wärmeexplosion“ handelt, sondern eher um eine Kettenreaktion in flüssiger Phase.

Der R u d o lf K o e p p A.-G., Chemische Fabrik (Oestrich im Rheingau), haben wir für freundliche Unterstützung dieser Untersuchung, dem Institut $\mathrm{Nik}$ ol a o s Ka ne llopoulos in Piräus für gewährte Gastfreundschaft zu danken. 